\title{
AKNA Is a Potential Prognostic Biomarker in Gastric Cancer and Function as a Tumor Suppressor by Modulating EMT-Related Pathways
}

\author{
Gang Wang $\mathbb{D}$, Dan Sun, Wenhui Li, and Yan Xin \\ Laboratory of Gastrointestinal Onco-Pathology, Cancer Institute, The First Affiliated Hospital of China Medical University, \\ Shenyang 110001, Liaoning Province, China
}

Correspondence should be addressed to Yan Xin; yxin@cmu.edu.cn

Received 20 January 2020; Revised 11 March 2020; Accepted 16 April 2020; Published 13 May 2020

Academic Editor: Nicola Cirillo

Copyright (c) 2020 Gang Wang et al. This is an open access article distributed under the Creative Commons Attribution License, which permits unrestricted use, distribution, and reproduction in any medium, provided the original work is properly cited.

\begin{abstract}
The AT-hook transcription factor, AKNA, is a nuclear protein that affects a few physiological and pathological processes including cancer. Here, we investigated the role of $A K N A$ in gastric cancer (GC). By using quantitative real-time polymerase chain reaction (qRT-PCR) and Western blot assays, AKNA was found deregulated in both GC cell lines and 32 paired GC tissues. Subsequently, Kaplan-Meier analysis and clinicopathological analysis were conducted using both $32 \mathrm{GC}$ cases' data above and RNA-Seq data of $A K N A$ in 354 GC patients and the corresponding clinical-pathological data obtained from The Cancer Genome Atlas (TCGA), and AKNA expression was found closely related to location, metastasis, and TNM staging of GC. Then, the potential molecular mechanisms of $A K N A$ in GC were explored by gene set enrichment analysis (GSEA), qRT-PCR, and Western blot assays. AKNA was found to be a hub gene related to homotypic cell to cell adhesion, regulation of cell to cell adhesion, leukocyte cell to cell adhesion, and regulation of T cell proliferation in GC. GO analysis revealed that AKNA involved in the regulation of epithelialmesenchymal transition (EMT)-related pathways including chemokine signaling pathway, cytokine to cytokine receptor interaction, cell adhesion molecules, and jak-stat signaling pathway in GC. To explore the regulation of AKNA expression, Targetscan and TargetMiner were used to predict the possible miRNA which targeted AKNA and found the expression of $A K N A$ was negatively correlated to miR-762 which could be sponged by circTRNC18. In conclusion, $A K N A$ could function as a tumor suppressor by modulating EMT-related pathways in GC. The expression of AKNA might be regulated by circTRNC18/miR-762 axis. AKNA could serve as a potential biomarker and an effective target for GC diagnosis and therapy.
\end{abstract}

\section{Introduction}

Gastric cancer (GC) is the fifth most frequent malignancies and the third most frequent cause of cancer-related death all over the world [1]. Despite great advances in the field of diagnosis and systemic treatment in recent years, the prognosis is unpleasant for GC patients, as the rapid progression to advanced stages and the peculiarity of highly metastatic for GC $[2,3]$. In the process of tumor distant metastasis, epithelial-mesenchymal transition (EMT) is a vital and initial molecular step [4]. Therefore, an improved understanding on the underlying mechanisms of the EMT involved in the process of GC metastasis is urgently needed for elucidating the development of relevant therapeutic approaches.
AKNA, also known as the AT-hook transcription factor, is a nuclear protein with AT-hook motif. Increasing evidences indicated crucial function of $A K N A$ might exert in multiple cancers. In cervical cancer, single-nucleotide polymorphisms (SNPs) make AKNA a susceptibility genetic factor [5]. AKNA directly binds the A/T-rich promoters regions of CD40 and CD40 ligand (CD40L) and coordinately regulates their expression, thereby activate antitumor immune response, while HPV E6, a cervical cancer-related oncoprotein, could downregulate $A K N A$ and lead to the progression of cancer $[6,7]$. Moreover, by using weighted gene coexpression network analysis (WGCNA), AKNA was found to be a hub gene of head and neck squamous cell carcinoma (HNSCC) which is related to the immune response [8]. 
Recently, Camargo et al. reported that AKNA could regulate EMT in neurogenesis [9]. As is well known that the expression of a certain mRNA could be regulated by circRNA which competitively sponge corresponding miRNA, circRNAs are a unique category of RNA molecules that were first identified in plant viruses in the 1970s [10]. Recently, they have aroused extensive attention as various circRNAs were found to play different roles in multiple diseases, especially in cancers [11, 12]. Growing evidences demonstrate that circRNAs usually regulate tumor progression and metastasis by affecting EMT [13]. CircTRNC18, a circRNA alias hsa_circ_0006772, which is transcripted from TNRC18 gene, was reported to negatively regulate trophoblast cell migration and EMT via regulating miR-762/Grhl2 axis in preeclampsia [14]. By using bioinformatics tools, we predicted that circTRNC18 could serve as a ceRNA of AKNA by competing sponge miR-762. However, the expression and regulatory role of circTRNC18/miR-762/ $A K N A$ axis in GC progression is not yet clear.

The present study was novel in demonstrating that $A K N A$, a potential target of miR-762, was deregulated in GC and was closely related to location, metastasis, and TNM staging of GC. GSEA analysis revealed AKNA could function as a hub gene of GC by regulating EMT-related pathways. AKNA might be regulated by circTRNC18/miR762 axis in GC. The present study provides a promising biomarker and a potential target for GC treatment.

\section{Materials and Methods}

2.1. Clinical Specimen Collection. There were 32 fresh primary GC and matched normal gastric epithelial tissues acquired from patients with GC undergoing resection in the First Affiliated Hospital of China Medical University. The samples were immediately collected and placed into RNasefree Eppendorf tubes after resection and put into liquid nitrogen for $5 \mathrm{~min}$, then were put in storage at $-80^{\circ} \mathrm{C}$ for further use. All primary tumor cases and matching normal tissues were verified by qualified pathologists. Permission was obtained from the ethics committee of the First Affiliated Hospital of China Medical University, and informed consent was obtained from patients.

2.2. Cell Culture. Five GC cell lines AGS, SGC-7901, BGC-823, MNK-45, HGC-27, and human immortalized normal gastric epithelial cells GES-1 were provided by Genechem Co., Ltd (Shanghai, China). All the cells were maintained and generated in RPMI 1640 (Gibco, USA) supplemented with 10\% fetal bovine serum (Hyclone, USA) and cultured at $37^{\circ} \mathrm{C}$ in $5 \% \mathrm{CO}_{2}$ atmosphere. Cells were collected when they reached the platform stage.

2.3. RNA Extraction and Real-Time PCR. RNA was extracted from GC cells and tissues using miRcute miRNA Kits (Tiangen Biotech Co., Ltd, Beijing, China) following the instructions. Then, $30 \mu \mathrm{l}$ diethylpyrocarbonate (DEPC)treated water was used to dissolve the extracted total RNA. The concentration and quality of RNA were determined using a NanoDrop spectrophotometer (Thermo Scientific, USA). Then, extracted RNA underwent reverse transcription using PrimeScript Master Mix (TaKaRa, Japan) for cDNA according to the manual instructions. The primers were constructed and synthesized by Sangon Biotech (Shanghai, China). The qRT-PCR assay was performed, and the expression levels were calculated using the $2-\triangle \triangle \mathrm{Ct}$ method. Sanger sequencing on the circTRNC18 qRT-PCR product was conducted by Sangon Biotech (Shanghai, China) to validate the specificity of the divergent primers and to confirm the back-splice junction sequence of circTRNC18 was consistent with that from circBase database. Glyceraldehyde-3phosphate dehydrogenase (GAPDH) was used as the internal reference for circTRNC18 and AKNA expression detection, and U6 snRNA was used as the internal reference for miR-762 expression detection. The reaction settings of the two-step PCR were as follows: $95^{\circ} \mathrm{C}$ for $30 \mathrm{~s} ; 40$ cycles of $95^{\circ} \mathrm{C}$ for $5 \mathrm{~s}$, annealing at $55^{\circ} \mathrm{C}$ for $30 \mathrm{~s}$; dissolving curve at $95^{\circ} \mathrm{C}$ for $15 \mathrm{~s}, 60^{\circ} \mathrm{C}$ for $30 \mathrm{~s}$, and $95^{\circ} \mathrm{C}$ for $15 \mathrm{~s}$. The primers were as follows: circTRNC18: forward: $5^{\prime}$-GGTG GCAGGGCTTGGAACGG-3' and reverse: $5^{\prime}$-GCCTTG TCTTGGAGCAGAGCTTC-3' ; miR-762: forward: GGGG CTGGGGCCGGGGC and reverse: universal downstream primer; AKNA: forward: 5' -GCACCAAGTCCGCAGCATC C-3' and reverse: $5^{\prime}$-CGCCATCCAGGTCTCCTCCA G-3'; GAPDH: forward: $5^{\prime}$-GAGTCAACGGATTTGGTCGT-3' and reverse: $5^{\prime}$-TTGATTTTGGAGGGATCTCG-3'; U6: forward: $5^{\prime}$-GGAACGATACAGAGAAGATTAGC-3' and reverse: $5^{\prime}$-TGGAACGCTTCACGAATTTGCG-3'.

2.4. Western Blot. Lysis buffer (Beyotime, China) was used to lyse GC tissues. The harvested protein concentration was quantified by microplate reader using a BCA protein kit (Beyotime, China). The proteins successively underwent electrophoresis, transferred membrane, blocked, and then incubated with primary antibody overnight using anti-AKNA (1:500, Proteintech, USA), anti-E-cadherin (1:1000, CST, USA), anti-N-cadherin ( $1: 1000$, CST, USA), anti-vimentin ( $1: 1000$, CST, USA), and GAPDH $(1: 1000$, Origene Co., Ltd., Beijing, China). After incubating with antimouse secondary antibody ( $1: 10000$, Origene Co., Ltd., Beijing, China), the blot analysis was conducted by an enhanced chemiluminescence system.

2.5. Bioinformatics Analysis. RNA-Seq data of AKNA in 354 GC patients and the corresponding clinical-pathological data were obtained from TCGA (https://cancergenome.nih.gov/) [15]. The survival curve was plotted using the Human Protein Atlas [16]. The potential molecular mechanisms of $A K N A$ in GC were explored by GSEA $[17,18]$, RNA-seq data of 354 GC patients in TCGA, and collection of annotated gene sets from molecular signatures database v7.0 were used to categorize enriched genes of $A K N A$-low expression group. Targetscan and TargetMiner were used to predict the possible miRNA which targeted AKNA [19, 20].

2.6. Statistical Analysis. All statistical analyses were conducted using SPSS25.0 (IBM, NY, USA). Data were presented as mean \pm standard error of the mean. The $\chi 2$ test, Student $t$ test, and one-way analysis of variance were used for 


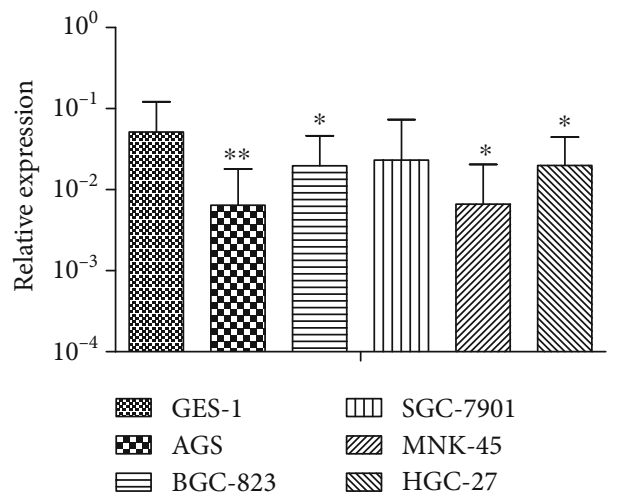

(a)

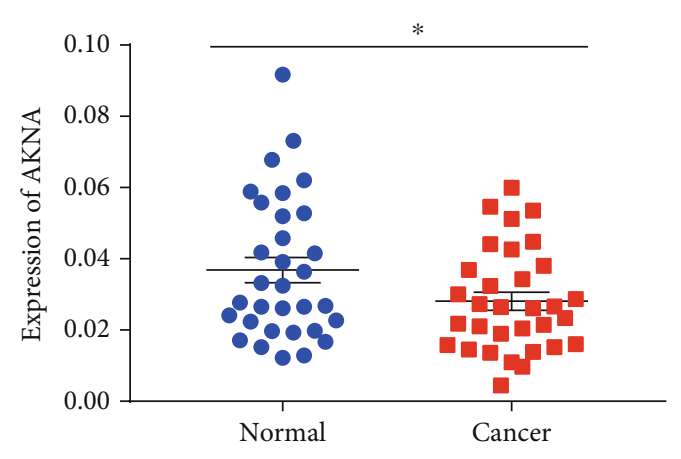

(b)

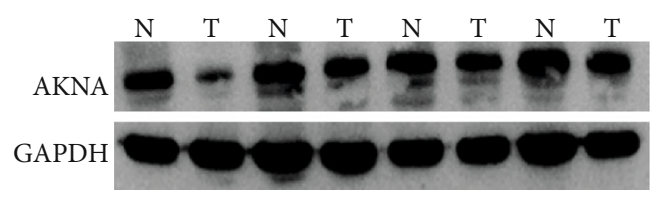

(c)
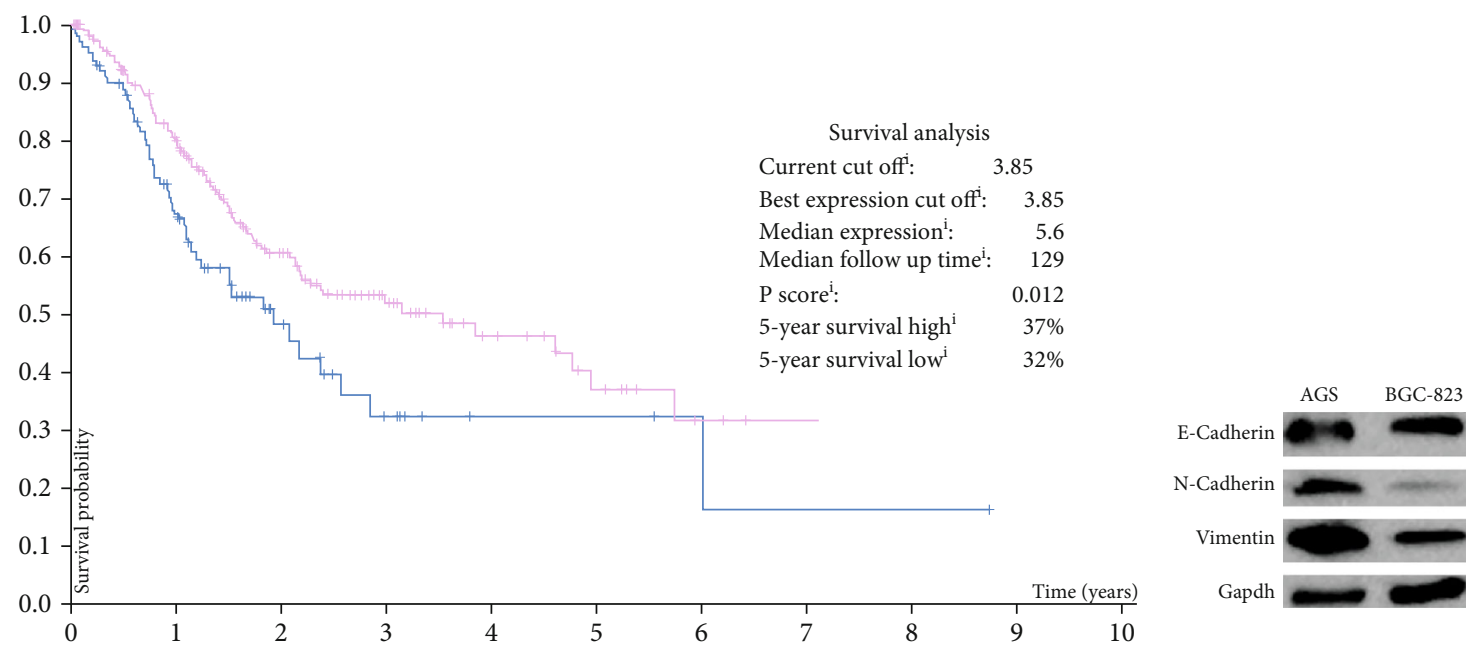

Low expression $(n=101)$

High expression $(n=253)$

FIgURE 1: The expression pattern of $A K N A$ in human GC. (a) The expression of AKNA in GC cell lines and GES-1 was detected by qRT-PCR. (b) AKNA expression in $32 \mathrm{GC}$ and paired normal tissues was detected by qRT-PCR. Data are means \pm SEM. $* P<0.05$. (c) Western blot assay was performed to determine AKNA expression in $32 \mathrm{GC}$ tissues and paired normal tissues. (d) Prognostic value of the expression of AKNA in 354 patients with GC in the TCGA database. (e) Western blot assay was performed to determine EMT-related markers expression in AGS and BGC-823 cells.

comparisons. A $P$ value less than 0.05 was considered statistically significant.

\section{Results}

3.1. Expression of AKNA Was Diminished in GC. qRT-PCR was performed to examine the expression of $A K N A$ in $\mathrm{GC}$ cell lines and 32 paired GC tissues. The results showed that the expression of $A K N A$ was significantly deregulated in GC cells compared to GES-1 (Figure 1(a)). In GC tissues, the expression of $A K N A$ was substantially lower than that in paired adjacent noncancerous tissues (Figure 1(b), $P<0.05)$. Furthermore, we conducted western blot to examine the expression of AKNA in 32 corresponding paired GC tissues in protein level, and a consistent expression was observed (Figure 1(c)).

3.2. The Expression Patterns of AKNA Were Related to the Prognosis of GC Patients and Metastasis of GC. Next, we valued the prognostic role of $A K N A$ in GC. By conducting Kaplan-Meier analysis, we found that GC patients with low AKNA expression usually had a worse survival time (5-year 
TABLE 1: The relationship between AKNA expression level and clinicopathological factors in 354 GC cases from the TCGA database.

\begin{tabular}{|c|c|c|c|c|c|}
\hline \multirow{2}{*}{ Characteristics } & \multirow{2}{*}{$N$} & \multicolumn{2}{|c|}{ Expression of $A K N A$} & \multirow{2}{*}{$\chi^{2}$} & \multirow{2}{*}{$P$} \\
\hline & & Low & High & & \\
\hline Gender & 354 & 101 & 253 & 0.108 & 0.742 \\
\hline Male & 229 & $64(27.9 \%)$ & 165 & & \\
\hline Female & 125 & $37(29.6 \%)$ & 88 & & \\
\hline Age(years) & 351 & 101 & 250 & 3.41 & 0.065 \\
\hline$\leq 65$ & 150 & $37(24.7 \%)$ & 113 & & \\
\hline$>65$ & 201 & $64(31.8 \%)$ & 137 & & \\
\hline Tumor sites & 340 & 96 & 244 & 13.256 & 0.004 \\
\hline Gastroesophageal junction & 39 & $16(41.0 \%)$ & 23 & & \\
\hline Cardiac & 45 & $19(42.2 \%)$ & 26 & & \\
\hline Fundus/body & 124 & $23(18.5 \%)$ & 101 & & \\
\hline Antrum & 132 & $38(28.8 \%)$ & 94 & & \\
\hline WHO's histological types & 103 & 24 & 79 & 3.005 & 0.391 \\
\hline \multicolumn{6}{|l|}{ Tubular Ade. } \\
\hline Moderately Diff. & 5 & $2(40.0 \%)$ & 3 & & \\
\hline Poorly Diff. & 68 & $18(26.5 \%)$ & 50 & & \\
\hline Mucinous Ade. & 11 & $1(9.1 \%)$ & 10 & & \\
\hline Signet ring cell car. & 19 & $3(15.8 \%)$ & 16 & & \\
\hline Lauren's types ${ }^{\mathrm{a}}$ & 233 & 65 & 168 & 1.668 & 0.196 \\
\hline Intestinal type & 161 & $49(30.4 \%)$ & 112 & & \\
\hline Diffuse type & 72 & $16(22.2 \%)$ & 56 & & \\
\hline Depth of invasion & 354 & 101 & 253 & 2.339 & 0.505 \\
\hline $\mathrm{T} 1$ & 18 & $7(38.9 \%)$ & 11 & & \\
\hline $\mathrm{T} 2$ & 62 & $20(32.3 \%)$ & 42 & & \\
\hline $\mathrm{T} 3$ & 12 & $2(16.7 \%)$ & 10 & & \\
\hline $\mathrm{T} 4$ & 262 & $72(27.5 \%)$ & 190 & & \\
\hline Ln metastasis & 318 & 83 & 235 & 2.375 & 0.498 \\
\hline No & 89 & $25(28.1 \%)$ & 64 & & \\
\hline N1 & 66 & $19(28.8 \%)$ & 47 & & \\
\hline $\mathrm{N} 2$ & 73 & $14(19.2 \%)$ & 59 & & \\
\hline N3 & 90 & $25(27.8 \%)$ & 65 & & \\
\hline Distant metastasis & 338 & 97 & 241 & 9.337 & 0.002 \\
\hline M0 & 315 & $84(26.7 \%)$ & 231 & & \\
\hline M1 & 23 & $13(56.5 \%)$ & 10 & & \\
\hline TNM staging & 306 & 82 & 224 & 15.012 & 0.002 \\
\hline I & 42 & $15(35.7 \%)$ & 27 & & \\
\hline II & 57 & $15(26.3 \%)$ & 42 & & \\
\hline III & 184 & 39 (21.2) & 145 & & \\
\hline IV & 23 & $13(56.5 \%)$ & 10 & & \\
\hline
\end{tabular}

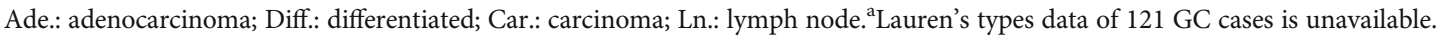

survival: low-32\% vs high $37 \%, P=0.012$ ) (Figure $1(\mathrm{~d})$ ). To further study the relationship between $A K N A$ expression and clinicopathological characters, clinicopathological analysis of 354 GC cases from TCGA database and 32 GC cases from our study were performed, respectively. Results showed that $A K N A$ expression in GC was closely related to location, metastasis, and TNM staging (Tables 1 and $2, P<0.05$ ). To further explore the possible mechanism, based on the qRT-PCR result, we detected the expression of several
EMT-related markers in AGS and BGC-823 cell lines by using the Western blot assay as BGC- 823 cell had a relatively higher expression of AKNA than that in AGS. BGC-823 revealed a lower expression of $\mathrm{N}$-cadherin and vimentin and a higher expression of E-cadherin than AGS (Figure 1(e)), which indicated that AKNA might inhibit metastasis by suppressing EMT. Consistent with results above, low expression of AKNA had a significantly worse prognosis than that of high AKNA expression in GC cases from the TCGA database. All 
TABLE 2: The relationship between AKNA expression level and clinicopathological factors in 32 GC cases.

\begin{tabular}{|c|c|c|c|c|c|}
\hline \multirow[b]{2}{*}{ Characteristics } & \multicolumn{3}{|c|}{ Expression of $A K N A$} & \multirow[b]{2}{*}{$x^{2}$} & \multirow[b]{2}{*}{$P$} \\
\hline & $N=32$ & $\begin{array}{c}\text { Low } \\
(N=16)\end{array}$ & $\begin{array}{c}\text { High } \\
(N=16)\end{array}$ & & \\
\hline Gender & & & & & $0.685 *$ \\
\hline Male & 24 & $13(54.2 \%)$ & 11 & & \\
\hline Female & 8 & $3(37.5 \%)$ & 5 & & \\
\hline Age(years) & & & & 4.800 & 0.028 \\
\hline$\leq 60$ & 12 & $9(75.0 \%)$ & 3 & & \\
\hline$>60$ & 20 & $7(35.0 \%)$ & 13 & & \\
\hline $\begin{array}{l}\text { Borrmann's } \\
\text { types }\end{array}$ & & & & & $0.433^{*}$ \\
\hline $\mathrm{I}+\mathrm{II}$ & 9 & $6(66.7 \%)$ & 3 & & \\
\hline III & 23 & $10(43.5 \%)$ & 13 & & \\
\hline Lauren's types & & & & 0.533 & 0.465 \\
\hline $\begin{array}{l}\text { Intestinal } \\
\text { type }\end{array}$ & 12 & $7(58.3 \%)$ & 5 & & \\
\hline Diffuse type & 20 & $9(45.0 \%)$ & 11 & & \\
\hline $\begin{array}{l}\text { Depth of } \\
\text { invasion }\end{array}$ & & & & 3.463 & 0.063 \\
\hline $\mathrm{T} 1 \sim \mathrm{T} 3$ & 11 & $3(27.3 \%)$ & 8 & & \\
\hline $\mathrm{T} 4$ & 21 & $13(61.9 \%)$ & 8 & & \\
\hline Ln metastasis & & & & 8.533 & 0.003 \\
\hline No & 12 & $2(16.7 \%)$ & 10 & & \\
\hline $\mathrm{N} 1 \sim \mathrm{N} 3$ & 20 & $14(70.0 \%)$ & 6 & & \\
\hline $\begin{array}{l}\text { Distant } \\
\text { metastasis }\end{array}$ & & & & & $1.000^{*}$ \\
\hline M0 & 31 & $15(48.4 \%)$ & 16 & & \\
\hline M1 & 1 & $1(100 \%)$ & 0 & & \\
\hline TNM staging & & & & 1.166 & 0.280 \\
\hline $\mathrm{I}+\mathrm{II}$ & 13 & $5(38.5 \%)$ & 8 & & \\
\hline III+IV & 19 & $11(57.9 \%)$ & 8 & & \\
\hline
\end{tabular}

*Fisher's exact test. Ln.: lymph node.

these results demonstrated that the expression of $A K N A$ was related to the GC patients' survival and might involve in the regulation of GC metastasis.

3.3. AKNA Involved in Regulation of Cell Adhesion and EMTRelated Pathways in GC. To further determine potential functions of $A K N A$ in GC progression, based on RNA-Seq data from TCGA, patients were divided into AKNA-high and $A K N A$-low groups. The association between $A K N A$ expression and related genes signatures were analyzed by GSEA. The genes signatures of homotypic cell to cell adhesion, regulation of cell to cell adhesion, leukocyte cell to cell adhesion, and regulation of $\mathrm{T}$ cell proliferation were all highly enriched in patients with $A K N A$ high expression, suggesting $A K N A$ was involved in these biological processes (Figure 2). KEGG pathway analysis of $A K N A$ via GSEA revealed that $A K N A$ mainly involved in chemokine signaling pathway, cytokine to cytokine receptor interaction, cell adhesion molecules, and jak-stat signaling pathway, which were closely related to EMT (Figure 3).
3.4. miR-762 Was Upregulated in GC and Might Target AKNA. TargetScan and TargetMiner software were used to predict the possible miRNA which could target AKNA. Then, miR-762 was chosen as it has a relatively high score on binding sites prediction with AKNA (Figures 4(a) and 4(b)). The expression and function of miR-762 in GC were not completely clear. Firstly, the expression level of miR-762 was assessed by qRT-PCR. Results showed that the expression of miR-762 was upregulated both in GC cells and tissues (Figures 4(c) and 4(d)). Then, the correlation coefficient was calculated, and a medium negative correlation between the expression of miR-762 and AKNA was observed in GC $(r=-0.555, P=0.001$; Figure $4(\mathrm{~g}))$, which indicated that miR-762 might target $A K N A$.

3.5. circTRNC18 Was Deregulated in GC and Was Negatively Correlated to $m i R-762$. CircRNAs customarily function as miRNA sponges to bind functional miRNAs. As miR-762 has been validated to be sponged by circTRNC18 by a dualluciferase system in preeclampsia [14], circTRNC18 was chosen for further research. The qRT-PCR assay was conducted to examine the expression of circTRNC18 in GC cells and tissues. The results showed a deregulated expression of circTRNC18 in GC (Figures 4(c) and 4(e)). The correlation analysis revealed a medium negative correlation between the expression of miR-762 and circTRNC18 in GC tissues $(r=-0.431, P=0.014$; Figure 4(f)). These data suggested that circTRNC18 might act as a tumor suppressor and circTRNC18 could serve as a molecular sponge for miR-762 in GC.

\section{Discussion}

Previous studies have revealed the crucial functions of $A K N A$ in multiple physiological and pathological processes such as development, immune function, inflammation, and cancer $[5,21,22]$. However, the role of AKNA in GC is not yet clear. In this study, by using qRT-PCR and bioinformatics analysis, we found $A K N A$ could be a promising biomarker of GC. Firstly, by using qRT-PCR and Western blot, we found AKNA was decreased in GC tissues in mRNA and protein levels, respectively. Manzo et al. conducted histology and immunohistochemistry (IHC) to evaluate the expression of AKNA in cervical biopsies of 12 cases of hysterectomy, which contains normal epithelium, cervicitis, and infiltrating squamous cell carcinoma (SCC), and found a substantial decrease of AKNA production in dysplasia area and SCC compared to normal cervical epithelium [7]. Our results were consistent with this trend.

Since the sample size of 32 is limited to conduct further analysis, the prognostic value of $A K N A$ was also evaluated through a dataset which contains 354 GC cases obtained from the TCGA database. A significant correlation between AKNA low expression and poor OS was found in GC. More interesting thing is that the expression level of $A K N A$ was correlated with tumor location, metastasis, and TNM staging. It could be inferred that $A K N A$ could be a prognostic biomarker for GC. 
Gene set 2369:GO_REGULATION_OF_HOMOTYPIC_CELL_CELL_ADHESION

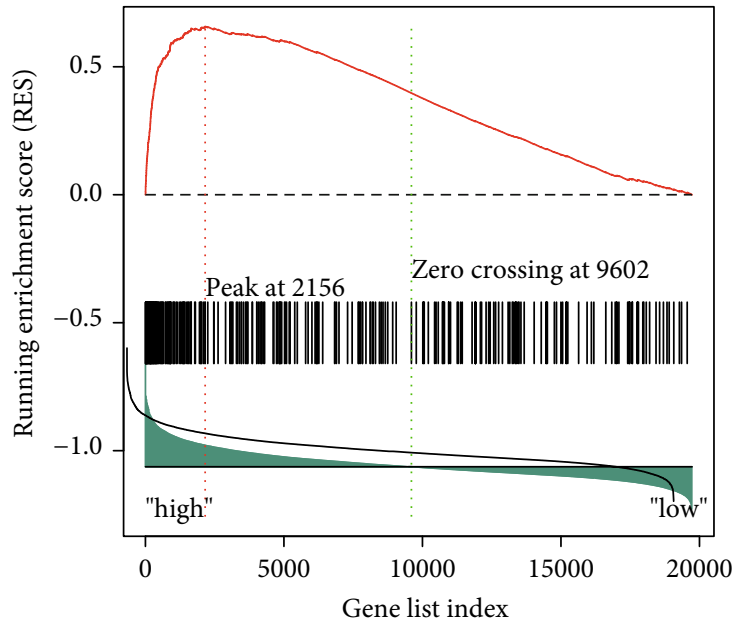

Number of genes: 19734 (in list), 296 (in gene set)

Gene set 2326 : GO_LEUKOCYTE_CELL_CELL_ADHESION

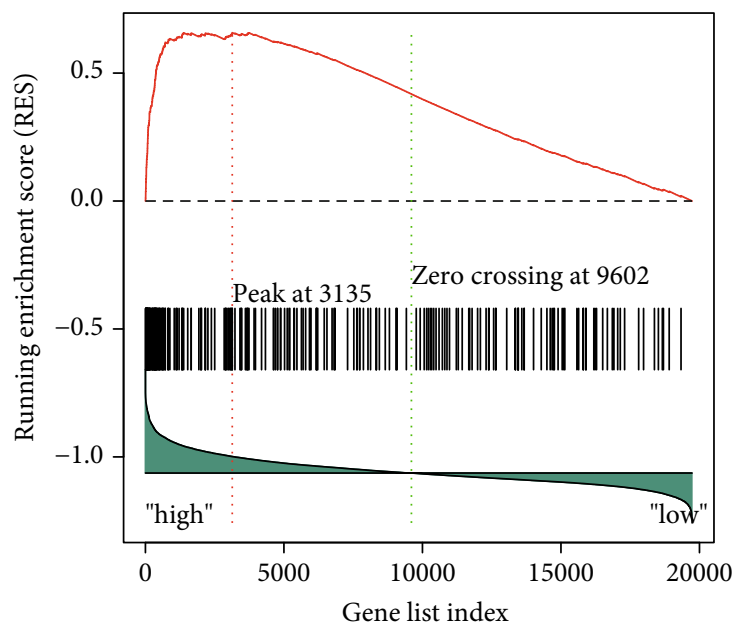

Number of genes: 19734 (in list), 254 (in gene set)
Gene set 2168 : GO_REGULATION_OF_CELL_CELL_ADHESION

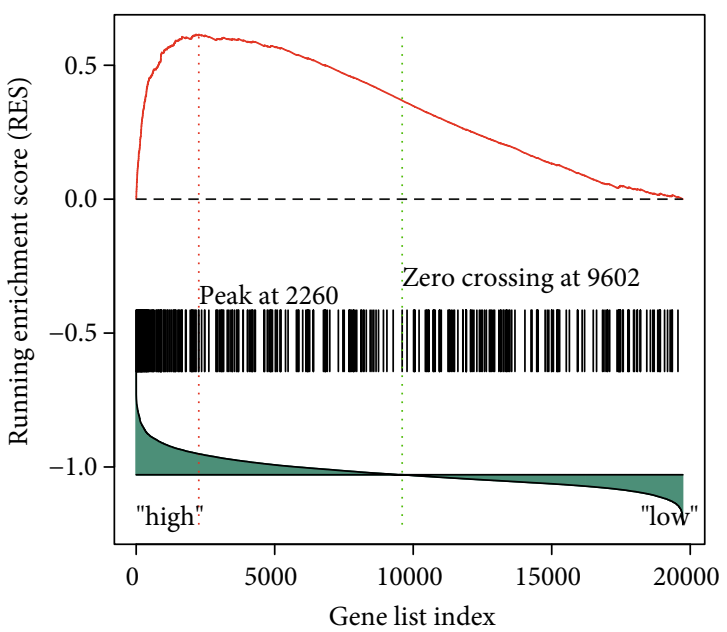

Number of genes: 19734 (in list), 296 (in gene set)

Gene set 1740 : GO_REGULATION_OF_T_CELL_PROLIFERATION

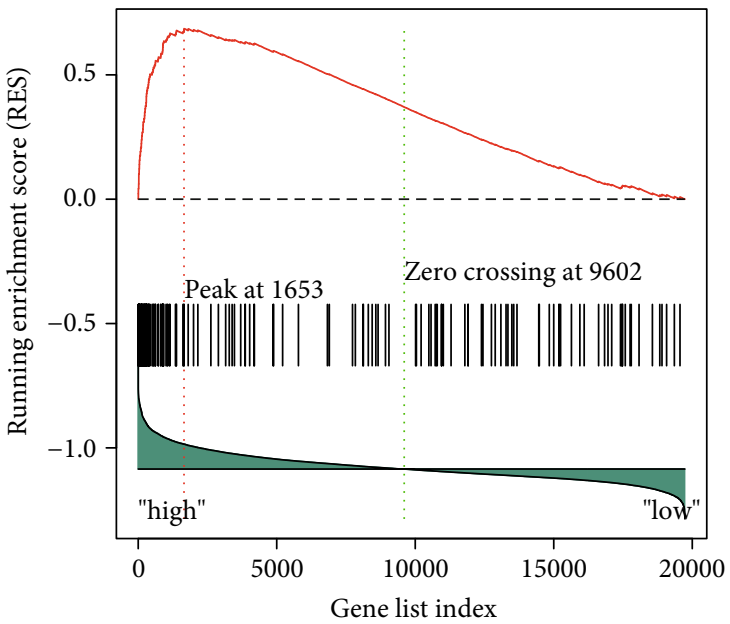

Number of genes: 19734 (in list), 145 (in gene set)

FIGURE 2: GO analysis of $A K N A$ in GC. The association between AKNA expression and related gene signatures was analyzed by GSEA followed by GO analysis.

In addition, our GSEA result revealed that $A K N A$ could regulate GC progression via various pathways like chemokine signaling pathway, cytokine to cytokine receptor interaction, cell adhesion molecules, and Janus kinase/signal transducer and activator of transcription (jak-stat) signaling pathway. Chemokines are a class of cytokines that act as signaling molecules, regulating immune and inflammatory responses. In the tumor microenvironment, chemokines were released to regulate cellular migration and cell-cell interactions, thereby mediate the balance between responses of antitumor and protumor. What is more, chemokines are also involved in other tumor progression processes such as tumor cell growth, angiogenesis, and tumor metastasis [23]. It is well known that jak-stat signaling is activated in multiple tumors including GC and implicated in tumor formation and metastatic progression, and cytokine IL-6 is a major activator of jak2/stat3 signaling [24]. Liu et al. reported that GH and inflammatory cytokines (TNF- $\alpha$, IL- $1 \beta$, and IL-6) expression were significantly downregulated after AKNA silencing [25]. Our results indicated that when the expression of $A K N A$ was dysregulated, the balance between responses of antitumor and protumor would be broken as a result of the abnormal activation of the chemokine signaling pathway and the jakstat signaling pathway.

Moreover, we found that dysregulated AKNA was related to cell to cell adhesion molecules in GO analysis. It is well known that the most important features of a malignant tumor are invasion and metastasis, and EMT is the initial step for tumor invasion and metastasis. The EMT is a process during which epithelial cells are gradually shifted into mesenchymal cells, which promotes the metastasis of tumor cells. During EMT, differentiated epithelial cells lose their apical-basal polarity and epithelial adhesion and acquire a myofibroblastic phenotype, with the change of multiple EMT-markers such as E-cadherin, N-cadherin, vimentin, and $\alpha$-smooth muscle actin, which is accompanied by 


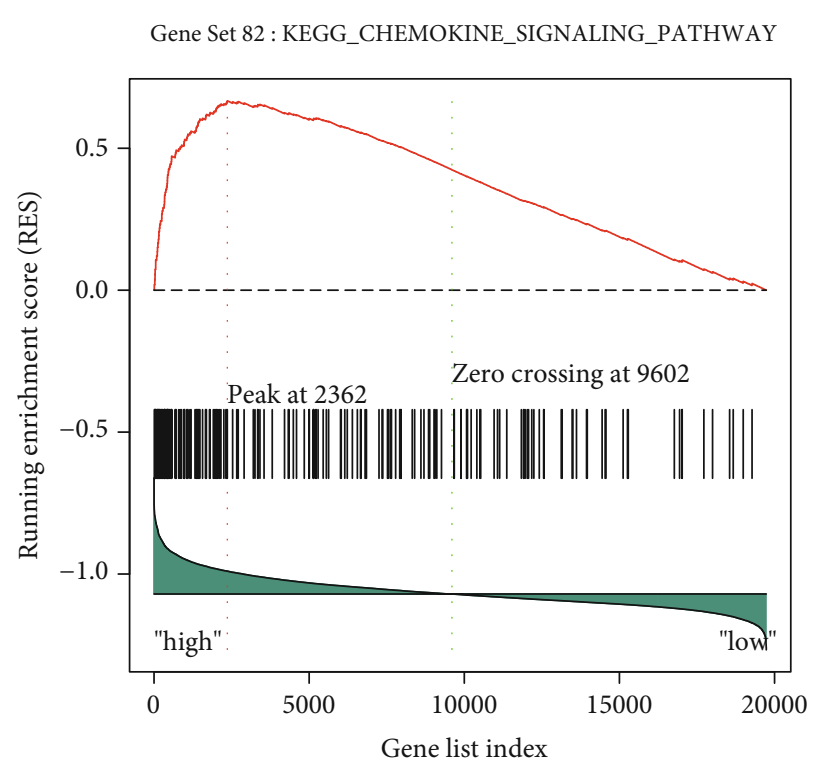

Number of genes: 19734 (in list), 186 (in gene set)

Gene set 107 : KEGG_CELL_ADHESION_MOLECULES_CAMS

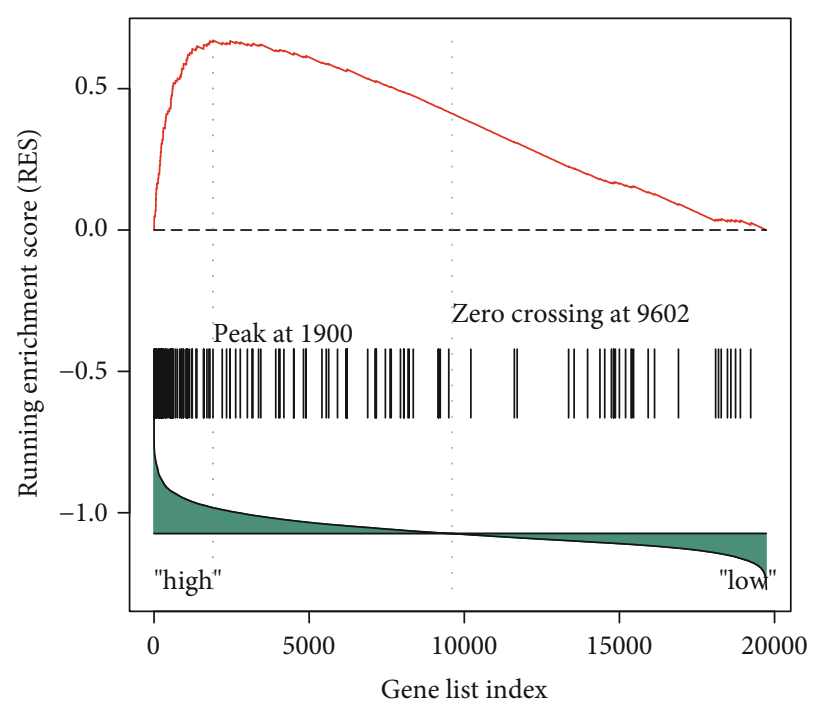

Number of genes: 19734 (in list), 131 (in gene set)
Gene set 81 : KEGG_CYTOKINE_CYTOKINE_RECEPTOR_INTERACTION

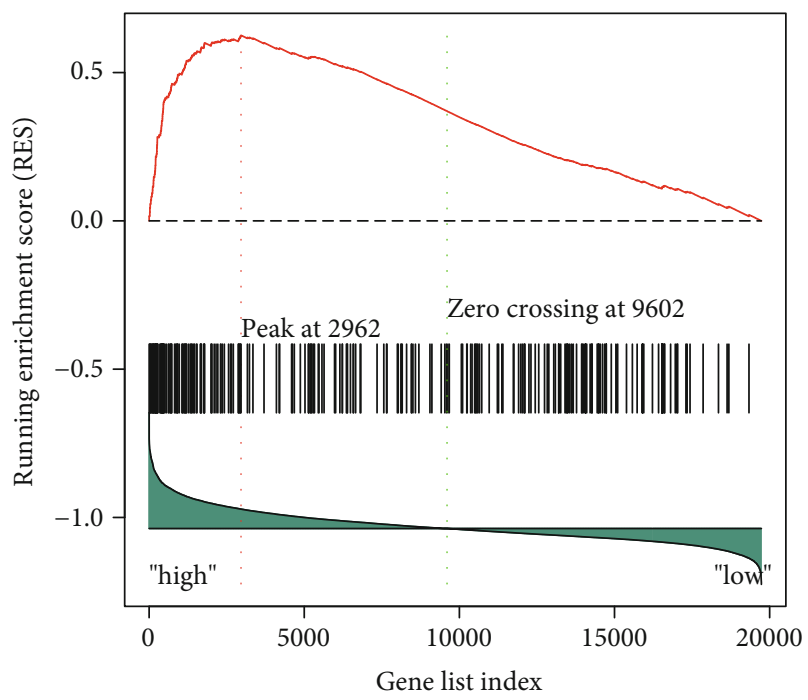

Number of genes: 19734 (in list), 258 (in gene set)

Gene set 118 : KEGG_CELL_ADHESION_MOLECULES_CAMS

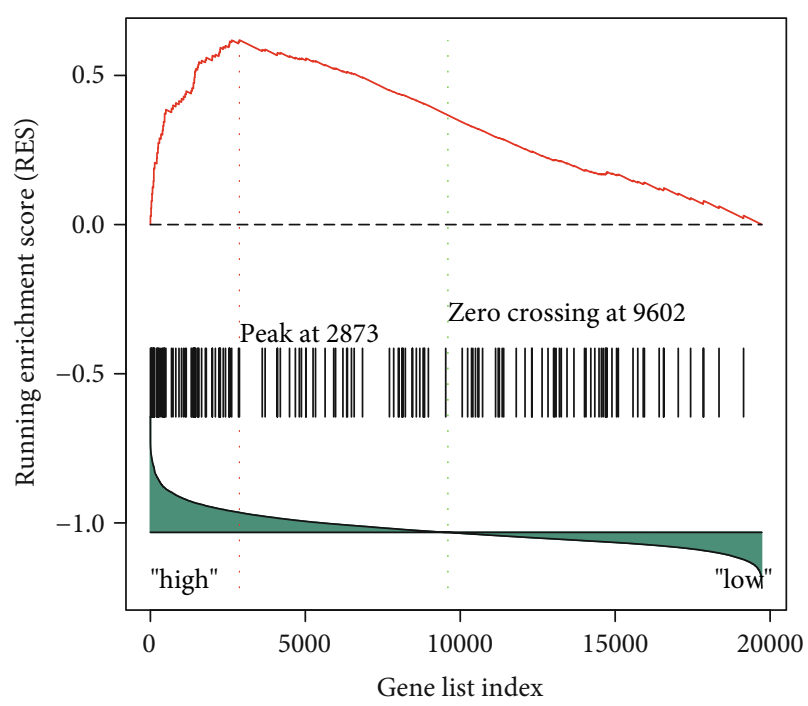

Number of genes: 19734 (in list), 151 (in gene set)

FIgURe 3: KEGG analysis of AKNA in GC. The association between AKNA expression and related gene signatures were analyzed by GSEA followed by KEGG analysis.

enhanced cell migration and invasion capabilities. Camargo et al. reported that knocking down of $A K N A$ in normal murine mammary gland epithelial cells during an EMT process induced by TGF $\beta 1$ led to ZO-1 tight junction protein and attenuated the rearrangement of actin fibres from the junctions to stress fibres, which indicated that the important role of AKNA in the regulation of cell adhesion in EMT [9]. Our Western blot assay result on EMT markers detection in AGS and BGC-823 cells was in line with this. It has been reported that in HNSCC, through WGCNA, AKNA was found to function as a hub gene that might involve in immune response, inflammatory response, and formation of the tumor microenvironment [8]. Our GSEA results revealed that the same effect of $A K N A$ might also exist in GC. What is more, we found that dysregulated $A K N A$ is probably related to distant metastasis of GC, and dysregulated $A K N A$ could lead to an attenuated cell adhesion via inducing EMT in GC. Considering that AKNA involves in signaling pathways related to the genesis and progression of GC, $A K N A$ could be a promising new therapeutic target for GC. However, as this conclusion is mainly drawn based on bioinformatic analysis, further experiments, including both in vitro and in vivo, are needed to explicit the potential role of $A K N A$ in GC.

Finally, we explored the molecular mechanism underlying the regulation of AKNA in GC. Through using bioinformatic 


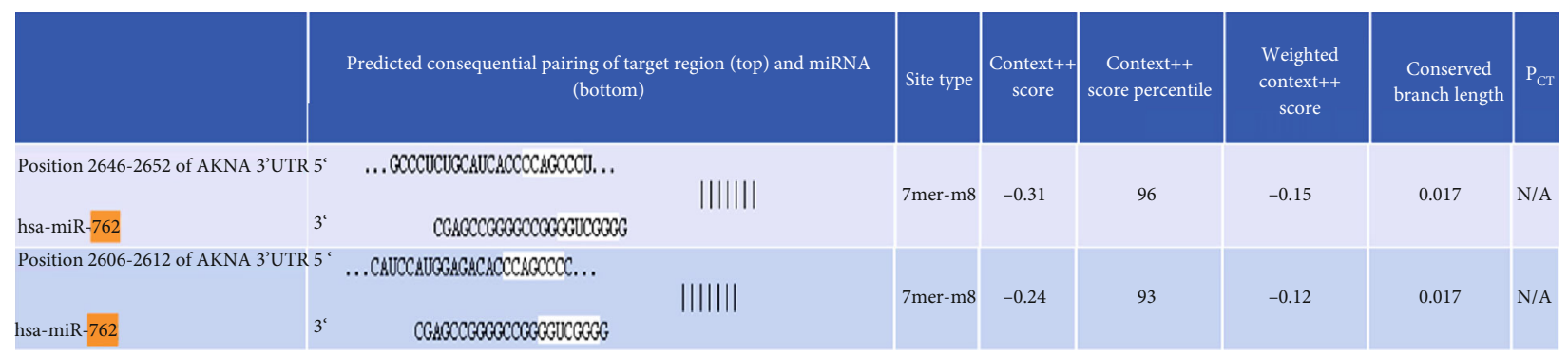

(a)

miRNA ID: $h s a-m i R-762$

miRNA Sequence: GGGGCUGGGGCCGGGGCCGAGC

\begin{tabular}{|c|c|c|c|c|c|c|c|c|c|}
\hline mRNA & Chromosome & 6 mer (count) & $\begin{array}{c}6 \text { mer } \\
\text { (position) }\end{array}$ & $\begin{array}{c}7 \text { mer-A1 } \\
\text { (count) }\end{array}$ & $\begin{array}{c}\text { 7mer-A1 } \\
\text { (position) }\end{array}$ & $\begin{array}{c}7 \text { mer-m8 } \\
\text { (count) }\end{array}$ & $\begin{array}{c}7 \text { mer-m8 } \\
\text { (position) }\end{array}$ & 8 mer (count) & $\begin{array}{c}8 \text { mer } \\
\text { (position) }\end{array}$ \\
\hline NM_030767 & chr9 & 11 & $\begin{array}{c}27152117 \\
11431003 \\
701663563 \\
450310182 \\
150\end{array}$ & - & - & 7 & $\begin{array}{c}27422652 \\
26122534 \\
24362131 \\
220\end{array}$ & - & - \\
\hline
\end{tabular}

(b)

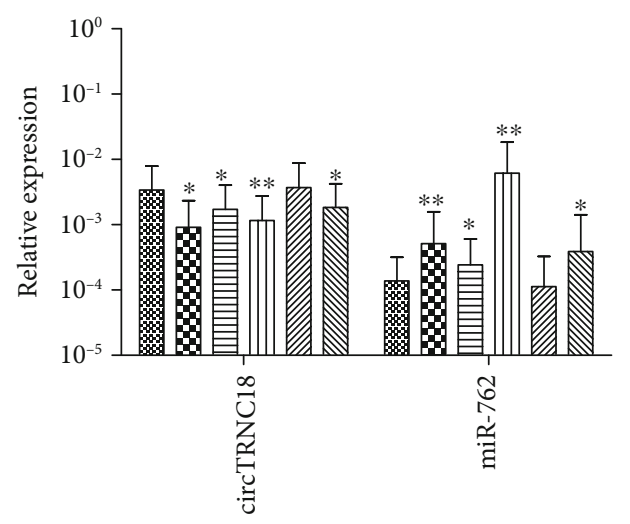

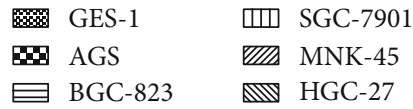

(c)

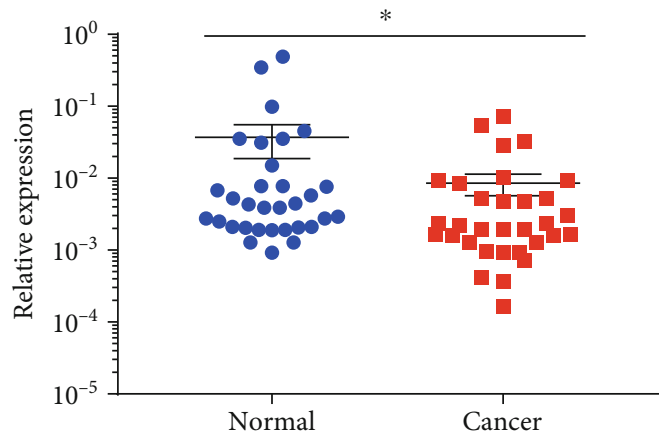

(e)

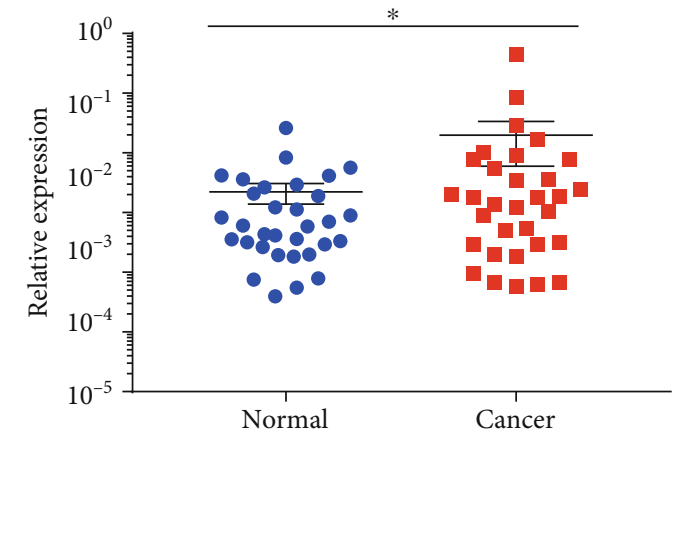

(d)

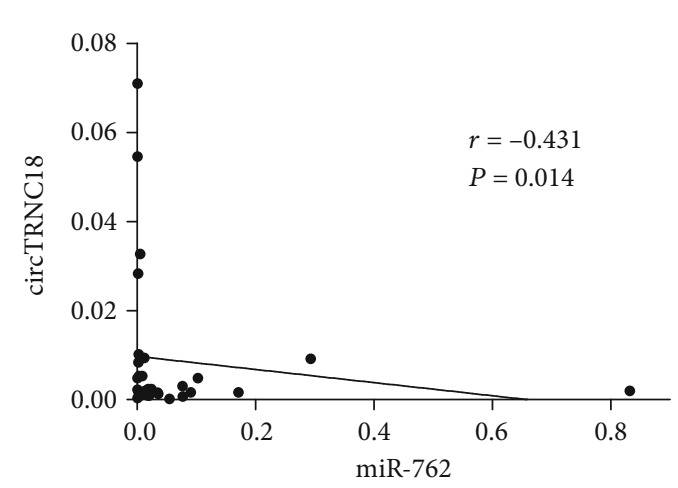

(f)

FIgURE 4: Continued. 


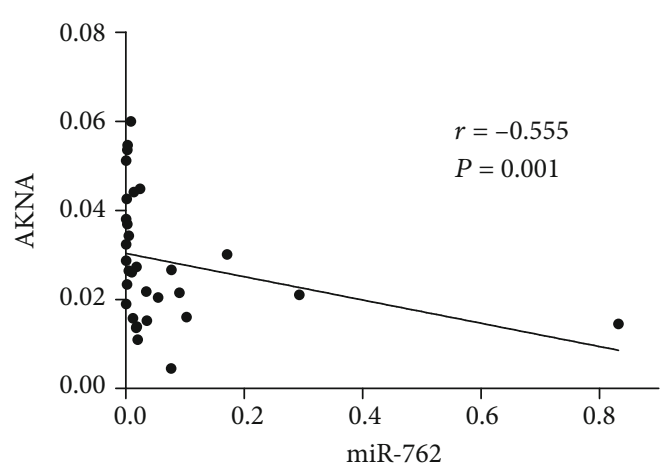

(g)

Figure 4: The correlation of AKNA and circTRNC18/miR-762 axis in GC. (a, b) One of the most possible miRNA targeted AKNA was predicted by Targetscan and TargetMiner. AKNA had multiple potential binding sites with miR-762. (c) The expression of circTRNC18 and miR-762 in GC cell lines and GES-1 was detected by qRT-PCR. Data are means \pm SEM. $* P<0.05, * * P<0.01$. (d) The expression of miR-762 in 32 GC and paired normal tissues was detected by qRT-PCR. Data are means \pm SEM. $* P<0.05$. (e) The expression of circTRNC18 in $32 \mathrm{GC}$ and paired normal tissues was detected by qRT-PCR. Data are means \pm SEM. $* P<0.05$. (f) Pearson's correlation coefficient was calculated between miR-762 and circTRNC18 expression in GC. $r=-0.431, P=0.014$. (g) Pearson's correlation coefficient was calculated between miR-762 and AKNA expression in GC. $r=-0.555, P=0.001$.

tools, we predicted the potential targets of $A K N A$ and chose the circTRNC18/miR-762 axis for the further research. In recent years, increasing studies have reported the remarkable role of noncoding RNAs (ncRNAs) in tumorigenesis and progression [26]. CircRNAs, a new class of ncRNAs, which are formed via back-splicing and have neither $5^{\prime}-3^{\prime}$ polarities nor polyadenylated tails structural, have gradually gained attention [27]. Increasing convincing evidences demonstrated that a large amount of circRNAs were dysregulated in multiple cancers including GC and could serve as sponges for miRNAs to regulate the expression of downstream targets. Du et al. reported that circ-PRMT5 was significantly upregulated in GC and could serve as miR-145 and miR-1304 "sponge," thereby upregulating expression of $m y c$, an oncogene, and promoting GC progression [28]. Dai et al. found that circGRAMD1B was downregulated in GC and confirmed that circGRAMD1B could serve as a tumor suppressor in GC by sponging miR-130a-3p via in vivo and in vitro experiments [29]. It was reported that circTRNC18 is negatively regulated trophoblast cell migration and EMT via regulating miR$762 / \mathrm{Grhl} 2$ axis in preeclampsia [14]; however, the role of circTRNC18 in GC is still unclear. Therefore, we firstly detected circTRNC18 in GC cells and tissues and demonstrated that circTRNC18 was decreased in GC. Moreover, we found miR-762 was upregulated in GC and negatively related to the expression of circTRNC18, which was consistent with the dual-luciferase reporter results of binding validation between circTRNC18 and miR-762 in the previous study [14], indicating that circTRNC18 might be a tumor suppressor in GC by sponging miR-762. By using bioinformatic tools and correlation analysis, we indicated that $A K N A$ might be the target of miR-762 in GC; however, there is further need for a dual-luciferase reporter assay to confirm their direct binding.

\section{Conclusion}

Taken together, AKNA is downregulated in GC. AKNA is a potential tumor suppressor, and it might function in GC through affecting EMT-related pathways including chemokine signaling pathway, cytokine to cytokine receptor interaction, cell adhesion molecules, and jak-stat signaling pathway. AKNA might be regulated by circTRNC18/miR762 axis. $A K N A$ could serve as a potential biomarker and an effective target for GC diagnosis and therapy.

\section{Data Availability}

The data used to support the findings of this study are available from the corresponding author upon request.

\section{Conflicts of Interest}

The authors have declared that no competing interest exists.

\section{Acknowledgments}

This study was supported by the National Natural Science Foundation of China (No. 81971584, No. 81071650); Special foundation for Science and Technology Program in Liaoning Province, China (2013225585); and Supporting Project for Climbing Scholars in Liaoning Provincial Universities, China (2009).

\section{References}

[1] F. Bray, J. Ferlay, I. Soerjomataram, R. L. Siegel, L. A. Torre, and A. Jemal, "Global cancer statistics 2018: GLOBOCAN estimates of incidence and mortality worldwide for 36 cancers in 185 countries," CA: A Cancer Journal for Clinicians, vol. 68, no. 6, pp. 394-424, 2018.

[2] A. Schlesinger-Raab, A. L. Mihaljevic, S. Egert et al., "Outcome of gastric cancer in the elderly: a population-based evaluation of the Munich Cancer registry," Gastric Cancer, vol. 19, no. 3, pp. 713-722, 2016.

[3] K. Tekesin, M. Emin Gunes, D. Tural et al., "Clinicopathological characteristics, prognosis and survival outcome of gastric 
cancer in young patients: a large cohort retrospective study," Journal of BUON, vol. 24, no. 2, pp. 672-678, 2019.

[4] W. Song, R. Mazzieri, T. Yang, and G. C. Gobe, “Translational significance for tumor metastasis of tumor-associated macrophages and epithelial-mesenchymal transition," Frontiers in Immunology, vol. 8, p. 1106, 2017.

[5] G. A. Martínez-Nava, K. Torres-Poveda, A. Lagunas-Martínez et al., "Cervical cancer-associated promoter polymorphism affects akna expression levels," Genes and Immunity, vol. 16, no. 1, pp. 43-53, 2015.

[6] A. Siddiqa, J. C. Sims-Mourtada, L. Guzman-Rojas et al., "Regulation of CD40 and CD40 ligand by the AT-hook transcription factor AKNA," Nature, vol. 410, no. 6826, pp. 383-387, 2001.

[7] J. Manzo-Merino, A. Lagunas-Martínez, C. Contreras-Ochoa et al., "The human papillomavirus (HPV) E6 oncoprotein regulates CD40 expression via the AT-hook transcription factor AKNA," Cancers, vol. 10, no. 12, p. 521, 2018.

[8] Y. Song, Y. Pan, and J. Liu, "The relevance between the immune response-related gene module and clinical traits in head and neck squamous cell carcinoma," Cancer Management and Research, vol. 11, pp. 7455-7472, 2019.

[9] G. Camargo Ortega, S. Falk, P. A. Johansson et al., “The centrosome protein AKNA regulates neurogenesis via microtubule organization," Nature, vol. 567, no. 7746, pp. 113-117, 2019.

[10] H. L. Sanger, G. Klotz, D. Riesner, H. J. Gross, and A. K. Kleinschmidt, "Viroids are single-stranded covalently closed circular RNA molecules existing as highly base-paired rodlike structures," Proceedings of the National Academy of Sciences of the United States of America, vol. 73, no. 11, pp. 3852-3856, 1976.

[11] Y. Wang, J. Liu, J. Ma et al., "Exosomal circRNAs: biogenesis, effect and application in human diseases," Molecular Cancer, vol. 18, no. 1, p. 116, 2019.

[12] L. Santer, C. Bar, and T. Thum, "Circular RNAs: a novel class of functional RNA molecules with a therapeutic perspective," Molecular Therapy, vol. 27, no. 8, pp. 1350-1363, 2019.

[13] B. Q. Shang, M. L. Li, H. Y. Quan et al., "Functional roles of circular RNAs during epithelial-to-mesenchymal transition," Molecular Cancer, vol. 18, no. 1, p. 138, 2019.

[14] X. Y. Shen, L. L. Zheng, J. Huang et al., "CircTRNC18 inhibits trophoblast cell migration and epithelial-mesenchymal transition by regulating miR-762/Grhl2 pathway in pre-eclampsia," RNA Biology, vol. 16, no. 11, pp. 1565-1573, 2019.

[15] K. Tomczak, P. Czerwinska, and M. Wiznerowicz, "The Cancer Genome Atlas (TCGA): an immeasurable source of knowledge," Współczesna Onkologia, vol. 19, no. 1A, pp. A68-A77, 2015.

[16] M. Uhlen, L. Fagerberg, B. M. Hallstrom et al., "Proteomics. Tissue-based map of the human proteome," Science, vol. 347, no. 6220, article 1260419, 2015.

[17] V. K. Mootha, C. M. Lindgren, K. F. Eriksson et al., "PGC1alpha-responsive genes involved in oxidative phosphorylation are coordinately downregulated in human diabetes," Nature Genetics, vol. 34, no. 3, pp. 267-273, 2003.

[18] A. Subramanian, P. Tamayo, V. K. Mootha et al., "Gene set enrichment analysis: a knowledge-based approach for interpreting genome-wide expression profiles," Proceedings of the National Academy of Sciences of the United States of America, vol. 102, no. 43, pp. 15545-15550, 2005.
[19] V. Agarwal, G. W. Bell, J. W. Nam, and D. P. Bartel, "Predicting effective microRNA target sites in mammalian mRNAs," eLife, vol. 4, 2015.

[20] S. Bandyopadhyay and R. Mitra, "TargetMiner: microRNA target prediction with systematic identification of tissuespecific negative examples," Bioinformatics, vol. 25, no. 20, pp. 2625-2631, 2009.

[21] W. Ma, B. Ortiz-Quintero, R. Rangel et al., "Coordinate activation of inflammatory gene networks, alveolar destruction and neonatal death in AKNA deficient mice," Cell Research, vol. 21, no. 11, pp. 1564-1577, 2011.

[22] A. R. Moliterno and L. M. Resar, "AKNA: another AT-hook transcription factor "hooking-up" with inflammation," Cell Research, vol. 21, no. 11, pp. 1528-1530, 2011.

[23] K. B. Goralski, A. E. Jackson, B. T. McKeown, and C. J. Sinal, "More than an adipokine: the complex roles of chemerin signaling in cancer," International Journal of Molecular Sciences, vol. 20, no. 19, p. 4778, 2019.

[24] X. Gao, J. Sun, C. Huang, X. Hu, N. Jiang, and C. Lu, "RNAimediated silencing of NOX4 inhibited the invasion of gastric cancer cells through JAK2/STAT3 signaling," American Journal of Translational Research, vol. 9, no. 10, pp. 4440-4449, 2017.

[25] X. Liu, D. Huang, P. Guo et al., "PKA/CREB and NF- $\kappa$ B pathway regulates AKNA transcription: a novel insight into T-2 toxin-induced inflammation and GH deficiency in GH3 cells," Toxicology, vol. 392, pp. 81-95, 2017.

[26] E. Anastasiadou, L. S. Jacob, and F. J. Slack, "Non-coding RNA networks in cancer," Nature Reviews Cancer, vol. 18, no. 1, pp. 5-18, 2018.

[27] S. P. Barrett and J. Salzman, "Circular RNAs: analysis, expression and potential functions," Development, vol. 143, no. 11, pp. 1838-1847, 2016.

[28] W. du, D. Li, X. Guo et al., "Circ-PRMT5 promotes gastric cancer progression by sponging miR-145 and miR-1304 to upregulate MYC," Artificial Cells, Nanomedicine, and Biotechnology, vol. 47, no. 1, pp. 4120-4130, 2019.

[29] X. Dai, X. Guo, J. Liu et al., "Circular RNA circGRAMD1B inhibits gastric cancer progression by sponging miR-130a-3p and regulating PTEN and p21 expression," Aging, vol. 11, no. 21, pp. 9689-9708, 2019. 